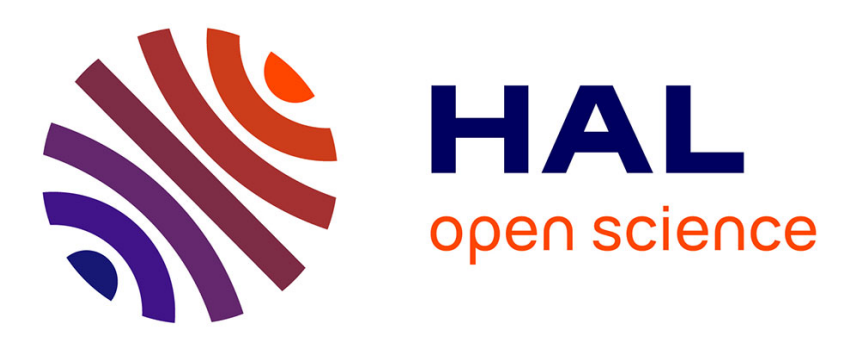

\title{
A probabilistic deformation of calculus of variations with constraints
}

Christian Léonard, Jean-Claude Zambrini

\section{To cite this version:}

Christian Léonard, Jean-Claude Zambrini. A probabilistic deformation of calculus of variations with constraints. Seminar on stochastic analysis, random fields and applications, VI, 2008, Ascona, Switzerland. pp.177-189. hal-00515417

\section{HAL Id: hal-00515417 \\ https://hal.science/hal-00515417}

Submitted on 6 Sep 2010

HAL is a multi-disciplinary open access archive for the deposit and dissemination of scientific research documents, whether they are published or not. The documents may come from teaching and research institutions in France or abroad, or from public or private research centers.
L'archive ouverte pluridisciplinaire HAL, est destinée au dépôt et à la diffusion de documents scientifiques de niveau recherche, publiés ou non, émanant des établissements d'enseignement et de recherche français ou étrangers, des laboratoires publics ou privés. 


\title{
A probabilistic deformation of calculus of vari- ations with constraints
}

\author{
Christian Léonard and Jean-Claude Zambrini
}

\begin{abstract}
In the framework of a probabilistic deformation of the classical calculus of variations, we consider the simplest problem of constraints, and solve it in two different ways. First by a pathwise argument in the line of Euclidean Quantum Mechanics. Second from an entropic (measure theoretic) perspective.
\end{abstract}

Mathematics Subject Classification (2000). Primary 49J55, 60F10, 60G57; Secondary 49L99, 49S05.

Keywords. Probabilistic calculus of variations, stochastic least action principle, conditional law of large numbers, relative entropy, Bernstein processes.

\section{Contents}

1. Introduction 1

2. Stochastic least action principle with final constraint 2

3. Random dynamical particles performing an unexpected final event $\quad 7$

References 11

\section{Introduction}

In classical calculus of variations, most concrete problems of optimization are accompanied with various kinds of constraints: isoperimetric, holonomic or not, etc. handled with the method of "Lagrange multipliers" (a misnomer, in fact, since it has been introduced by Euler), see for example [7].

In [3], we presented what can be regarded as a probabilistic deformation of the classical calculus of variations. Its basic principle is to deform in a minimal way the ideas and tools of the classical theory, so that those become compatible with the very irregular paths of diffusion processes. Since then, it has been shown that the same method holds for a much wider class of stochastic processes [11]. 
By minimal deformation, we mean one involving exclusively the regularizations associated with the existence of the underlying probability measures on path spaces. By construction, our variational framework depends on a positive constant $\hbar$. At the ("classical") limit where this constant vanishes, those paths become smooth and any claim should reduce to a statement of classical calculus of variations.

We are going to consider a problem of constraint in this probabilistic context, from a dual perspective. First, along the original line of [3], namely in the deformed variational perspective. Then, in a measure theoretic perspective, with a strong entropic flavor. It has been known, indeed, for a long time that the diffusions critical points of our regularizartion functionals belong to special time-symmetric class (Bernstein's reciprocal class) which can be naturally described in this way, see for instance $[2,6,9]$. As a matter of fact, the second perspective makes, in a way, clearer the status of Lagrange multipliers in our deformed calculus of variations.

In the present paper, one mostly presents the main ideas without focusing to precise assumptions and detailed proofs. The constraint considered here (prescribed final condition) is the simplest one in a hierarchy of manageable constraints. They will be systematically explored in future publications with a complete mathematical treatment.

\section{Notation}

For any measurable set $A, \mathcal{P}(A)$ denotes the set of all probability measures on $A$. The space $\Omega=C\left([0, T], \mathbb{R}^{d}\right)$ of all continuous paths from the time interval $[0, T]$ to the configuration space $\mathbb{R}^{d}$ is equipped with its usual $\sigma$-field. The canonical process on $\Omega$ is $\left(X_{t}\right)_{0 \leq t \leq T}$.

One denotes by $P_{t}=X_{t} \# P$ the image law of $X_{t}$ under $P \in \mathcal{P}(\Omega)$ and $P_{t y}=$ $P\left(\cdot \mid X_{t}=y\right)$.

\section{Stochastic least action principle with final constraint}

Let us consider the following action functional on $\mathcal{P}(\Omega)$

$$
J(P)=E^{P} \int_{0}^{T} L\left(X_{t}, D^{P} X_{t}\right) d t+E^{P} S\left(X_{T}\right), \quad P \in \mathcal{D}_{J}
$$

defined on a domain $\mathcal{D}_{J}$ of diffusion processes, with measures $P \in \mathcal{P}(\Omega)$, solving stochastic differential equations (SDE) of the form

$$
\left\{\begin{array}{l}
d X_{t}=b\left(X_{t}, t\right) d t+\sqrt{\hbar} d W_{t} \\
X_{0}=x
\end{array}\right.
$$

where $W$ is the Wiener process, $b$ a Markov measurable "drift" and $\hbar$ a positive constant, our deformation parameter. It should be noted that $b=b^{P}$ can be regarded as a parametrization of $P$ in $\mathcal{D}_{J}$. In definition (2.1),

$$
L:(q, v) \in \mathbb{R}^{d} \times \mathbb{R}^{d} \mapsto L(q, v) \in \mathbb{R}
$$


is called the Lagrangian of $J, D^{P}$ stands for the partial differential operator of parabolic type associated with the diffusion law $P$, defined on smooth (real valued) functions $\phi(q, t)$ by

$$
D^{P} \phi=\partial_{t} \phi+b^{P}(q, t) \partial_{q} \phi+\frac{\hbar}{2} \partial_{q}^{2} \phi
$$

In our "deformation perspective", its proper interpretation is given by the following regularizations (or "deformation") of time derivative of $\phi$ along the solution of $(2.2)$ :

$$
D^{P} \phi\left(X_{t}, t\right)=\lim _{\Delta t \downarrow 0} E^{P}\left[\frac{\phi\left(X_{t+\Delta t}, t+\Delta t\right)-\phi\left(X_{t}, t\right)}{\Delta t} \mid X_{t}\right]
$$

In particular, when $\phi(q, t)=q:=\operatorname{pr}_{q}(q, t)$, with some abuse of notation $D^{P} \operatorname{pr}_{q}\left(X_{t}, t\right)$ reduces to

$$
D^{P} X_{t}=b^{P}\left(X_{t}, t\right)
$$

the second (random) variable of $L$, regarded as independent of the first one. The existence of such a space of independent variables, in this context, is in itself an interesting geometrical problem which is solved by a deformation of its classical solution, i.e. of classical Contact Geometry. This aspect will not be elaborated here (for a glimpse, see [10, 14]).

Let us consider the following "constrained problem": Find the critical points of the action functional (2.1) in the class of diffusions (2.2) such that, in addition, the following final constraint holds:

$$
E^{P} N\left(X_{T}\right)=0
$$

for a given measurable function $N$ satisfying some integrability conditions.

Now, we introduce, in complete analogy with the classical case, the new, unconstrained, action functional

$$
J^{\lambda}(P)=E^{P} \int_{0}^{T} L\left(X_{t}, D^{P} X_{t}\right) d t+E^{P}(S-\lambda N)\left(X_{T}\right)
$$

for $\lambda \in \mathbb{R}$ the Lagrange multiplier.

Then, as expected, the claim is the following

Theorem 2.1. If, for any $\lambda \in \mathbb{R}$ (or $\lambda$ in an interval) we can find $P^{\lambda}$ in the class (2.2) critical point of the unconstrained action functional (2.5) and if there is a $\lambda_{o}$ (in the same interval) for which the constraint (2.4) is satisfied:

$$
E^{P^{\lambda_{o}}} N\left(X_{T}\right)=0
$$

then $P^{\lambda_{o}}$ is a critical point of the constrained problem and the process associated with $P=P^{\lambda_{o}}$ solves the stochastic Euler-Lagrange equation:

$$
\left[D^{P} \partial_{v} L-\partial_{q} L\right]\left(X_{t}, D^{P} X_{t}\right)=0,0<t<T, \quad P-a . s .
$$

with

$$
\left\{\begin{array}{l}
\partial_{v} L\left(X_{T}, D^{P} X_{T}\right)=-\nabla\left(S-\lambda_{o} N\right)\left(X_{T}\right) \quad \text { P-a.s. } \\
X_{0}=x
\end{array}\right.
$$


Idea of proof. Let us apply to the unconstrained action $J^{\lambda}$ the method of [3] (see also $[1, \S 6.2])$ to compute its variation at $P$ in a direction $\delta P$, for $\delta P$ a signed measure such that $P+\delta P$ is a Markov probability measure absolutely continuous with respect to $P$. By Girsanov's theorem, this implies that there exists a bounded variation random process $\delta X$ such that the image measure $(X+\delta X) \# P=P+\delta P$. It is enough to differentiate $J^{\lambda}$ in the directions $\delta X$ with sample paths in the Cameron-Martin space. This gives

$$
\begin{gathered}
\left\langle\nabla J^{\lambda}(P) \mid \delta P\right\rangle:=\left.\frac{d}{d \epsilon}\right|_{\epsilon=0}\left\{E^{P} \int_{0}^{T} L\left((X+\epsilon \delta X)_{t}, D^{P}(X+\epsilon \delta X)_{t}\right) d t\right. \\
\left.+E^{P}(S-\lambda N)\left(X_{T}+\epsilon \delta X_{T}\right)\right\} \\
=E^{P} \int_{0}^{T}\left(\partial_{q} L\left(X_{t}, D^{P} X_{t}\right) \delta X_{t}+\partial_{v} L\left(X_{t}, D^{P} X_{t}\right) D^{P} \delta X_{t}\right) d t \\
+E^{P}\left[(\nabla S-\lambda \nabla N)\left(X_{T}\right) \delta X_{T}\right]
\end{gathered}
$$

For the second term under the integral, we appeal to Itô's product formula for $\delta X$ of bounded variation:

$$
D^{P}\left[\partial_{v} L\left(X_{t}, D^{P} X_{t}\right) \delta X_{t}\right]=D^{P}\left[\partial_{v} L\left(X_{t}, D^{P} X_{t}\right)\right] \delta X_{t}+\partial_{v} L\left(X_{t}, D^{P} X_{t}\right) D^{P}\left[\delta X_{t}\right]
$$

Since the differential meaning of $(2.3)$ is $E^{P}\left[D^{P} \phi\left(X_{t}, t\right) d t\right]=E^{P}\left[d \phi\left(X_{t}, t\right),\right]$ the r.h.s. of (2.9) reduces to

$$
\begin{aligned}
E^{P} \int_{0}^{T}\left[\partial_{q} L-D^{P} \partial_{v} L\right] & \left(X_{t}, D^{P} X_{t}\right) \delta X_{t} d t \\
& +E^{P}\left\{\left[\partial_{v} L\left(X_{T}, D^{P} X_{T}\right)+\nabla(S-\lambda N)\left(X_{T}\right)\right] \delta X_{T}\right\}
\end{aligned}
$$

A diffusion $P^{\lambda}$ in the class $(2.2)$ is extremal (or critical) for the functional $J^{\lambda}$ if $\left\langle\nabla J^{\lambda}(P) \mid \delta P\right\rangle=0$ for any admissible $\delta P$ in the Cameron-Martin subspace. By (2.10) this means that, $P^{\lambda}$-a.s.

$$
\begin{aligned}
& {\left[\partial_{q} L-D^{P^{\lambda}}\left(\partial_{v} L\right)\right]\left(X_{t}, D^{P^{\lambda}} X_{t}\right)=0 \text { and }} \\
& \partial_{v} L\left(X_{T}, D^{P^{\lambda}} X_{T}\right)+\nabla(S-\lambda N)\left(X_{T}\right)=0 .
\end{aligned}
$$

In particular, if $\lambda=\lambda_{o}$ then the final constraint (2.6) is satisfied, as well as, by definition, for any diffusion satisfying (2.4) whose boundary term $N$ is irrelevant to the action. This means that $P^{\lambda_{o}}$ is critical for the above constrained problem and solves (2.7) and (2.8).

This argument is along the line of a verification theorem in the sense of stochastic optimal control theory (see [5] for instance). Let us see how it works in practice. 
Consider a typical example suggested by "Euclidean Quantum Mechanics" [1], namely the action functional

$$
J(P)=E^{P} \int_{s}^{T}\left\{\frac{1}{2}\left|D^{P} X_{t}\right|^{2}+\frac{1}{2} X_{t}^{2}\right\} d t
$$

where instead of a zero initial time, as before, it will be useful to pick any time $s<T$.

We are looking for a critical point of $J$ in the class of diffusions (2.2) such that

$$
E_{s x}^{P}\left[X_{T}^{2}-m^{2}\right]=0, \quad \text { for } m \text { a given constant. }
$$

According to the theorem, we need first to find, for any $\lambda \in \mathbb{R}$, the critical point of the (unconstrained) functional

$$
J^{\lambda}(P)=E_{s x}^{P} \int_{s}^{T}\left\{\frac{1}{2}\left|D^{P} X_{t}\right|^{2}+\frac{1}{2} X_{t}^{2}\right\} d t-\lambda E_{s x}^{P}\left[X_{T}^{2}-m^{2}\right]
$$

This is an action of the form (6.25) in [1], for a quadratic potential $V(q)=\frac{1}{2} q^{2}$ and a final value $S_{L}(q, T):=-\lambda\left(q^{2}-m^{2}\right)$.

A key point of the method advocated in [1] (dating back, in fact, to the mid-eighties, see [13] and references in [3]) is the following change of variables

$$
S_{L}(q, T)=-\hbar \log \eta_{T}(q)
$$

Here, $\eta_{T}(q)=e^{\frac{\lambda}{\hbar}\left(q^{2}-m^{2}\right)}$. Indeed, the local characteristics of the diffusion $P^{\lambda}$, critical for $J^{\lambda}$, will be provided by the (positive) solution of the PDE with final boundary condition

and "Hamiltonian"

$$
\begin{cases}\hbar \partial_{t} \eta & =\mathcal{H} \eta, \quad t<T \\ \eta(q, T) & =\eta_{T}(q)\end{cases}
$$

$$
\mathcal{H}=-\frac{\hbar^{2}}{2} \partial_{q}^{2}+V(q)
$$

This solution is given explicitly, in terms of the ("Mehler") integral kernel

$$
h(q, T-t, y)=\operatorname{kernel}\left\{e^{-\frac{T-t}{\hbar} \mathcal{H}}\right\}(q, y)
$$

by

$$
\eta(q, t)=\int_{\mathbb{R}} h(q, T-t, y) \eta_{T}(y) d y
$$

The critical process $P^{\lambda}$ of $J^{\lambda}$ solves a SDE of the form (2.2) for the drift

$$
b^{\lambda}(q, t)=-\nabla S_{L}(q, t):=\hbar \nabla \log \eta(q, t)=-\left[\frac{\sinh (T-t)+2 \lambda \cosh (T-t)}{\cosh (T-t)+2 \lambda \sinh (T-t)}\right] q
$$

Now, let us look for a $\lambda_{o} \in \mathbb{R}$ such that the constraint

$$
E_{s x}^{P^{\lambda}}\left[\left(X_{T}^{\lambda_{o}}\right)^{2}\right]=m^{2}
$$

is satisfied. 
As observed in the introduction, the structure of the diffusions $P^{\lambda}$ critical for action functionals such as $J^{\lambda}$ is very special. Their probability density, for any fixed $\mathcal{H}$, is of the Bernstein reciprocal form $[3,1]$

$$
P\left(X_{t} \in A\right)=\int_{A} \eta^{*}(q, t) \eta(q, t) d q
$$

with $t$ in the existence interval of $P, A$ a Borel set and $\eta^{*}$ a positive solution of the equation adjoint to $(2.11)$ with respect to the time parameter $t$ :

$$
\left\{\begin{array}{l}
-\hbar \partial_{t} \eta^{*}=\mathcal{H} \eta^{*}, \quad s \leq t \leq T \\
\eta^{*}(q, s)=\eta_{s}^{*}(q)
\end{array}\right.
$$

The product form (2.16) is, in fact, the key one for the relation with the entropic approach of $\S 3$ (cf. Conclusion).

Our constraint expectation (2.15) is a conditional one, knowing that $X_{s}=x$. This means that $\eta_{s}^{*}(q) d q=\delta_{x}(d q)$. The relevant normalized probability density of $P_{t}^{\lambda}$ is therefore of the special product form

$$
\mathcal{N}^{-1} h(x, t-s, q) \eta(q, t) d q
$$

with $\eta$ given by (2.13) and a normalizing factor $\mathcal{N}=\eta(x, s)$.

For $\eta_{T}(q)$ as prescribed before, the l.h.s. of (2.15) means

$$
E_{s x}^{P^{\lambda_{o}}}\left[\left(X_{T}\right)^{2}\right]=\frac{1}{2 \alpha}\left(1+\frac{\beta^{2}}{2 \alpha}\right)
$$

where $\alpha=\hbar^{-1}\left(\frac{1}{2} \operatorname{coth}(T-s)-\lambda_{o}\right), \beta=(\hbar \sinh (T-s))^{-1} x$.

The constraint (2.15) becomes equivalent to a quadratic polynomial in $\lambda_{o}$, whose coefficient depend on $m, T, x$ and $s$. So $P^{\lambda_{o}}$ with drift $b^{\lambda_{o}}$ of the form (2.14) is a solution of the constrained problem and $P=P^{\lambda_{o}}$ (or, better, its associated diffusion) solves

$$
\left\{\begin{array}{l}
D D^{P} X_{t}=X_{t}, \quad \forall s \leq t \leq T ; \quad P \text {-a.s. } \\
X_{s}=x ; \\
D^{P} X_{T}=b^{\lambda_{o}}\left(X_{T}, T\right)
\end{array}\right.
$$

which is a stochastic Euler-Lagrange equation with boundary conditions, for the given Lagrangian. As expected on a classical basis, an endpoint constraint such as (2.15) does not affect the dynamics of the extremal process of our stochastic action functional (given by (2.18)), but only the boundary conditions it should satisfy.

Let us observe that, in the perspective of the above calculus of variations, it is natural to regard $X_{t}$ as a (random) variable subjected to a variational procedure.

In the next $\S$, it will be more appropriate to consider the process as a fixed canonical one and the probability laws as the true variables of optimization. 


\section{Random dynamical particles performing an unexpected final event}

In 1932, Erwin Schrödinger addressed (in [12]) a problem of Brownian particles performing an unexpected event. His answer founded what we call, since the mid eighties, Euclidean Quantum Mechanics (cf [3], [13], for instance).

The problem of classical statistical physics is the following one. Suppose that you observe at time $t=0$, a large number $n$ of independent Brownian particles with some configuration distribution on $\mathbb{R}^{d}$ close to the probability measure $\mu$ and that at some later time $T$ you observe that their distribution $\nu$ differs significantly from the expected distribution $\mu * \gamma_{T}$ : the convolution of the initial state $\mu$ with the centered Gaussian measure with variance $T$. What is the most probable evolution of the whole system between these two states?

A modern answer to this problem is in terms of large deviations when $n$ tends to infinity of the empirical measure

$$
L_{n}^{Y}:=\frac{1}{n} \sum_{i=1}^{n} \delta_{Y^{i}}
$$

for the $n$ random paths $Y^{1}, \ldots, Y^{n}\left(\delta_{Y}\right.$ is the Dirac measure at $Y: t \in[0, T] \mapsto$ $\left.Y_{t} \in \mathbb{R}^{d}\right)$, see [6]. Note that $L_{n}^{Y}$ is a random element of $\mathcal{P}(\Omega)$. In this section, inspired by the above example, we address a similar problem where the $n$ particles are no longer Brownian but are independent and identically distributed with the law

$$
R^{V}:=z_{V}^{-1} \exp \left(-\frac{1}{\hbar} \int_{0}^{T} V\left(X_{t}\right) d t\right) R
$$

where $R$ is the Wiener measure with variance $\hbar$ and initial law $X_{0} \# R=\mu=\delta_{x}$, i.e. the law of (2.2) with $b=0$, and $V$ some real function such that

$$
z_{V}:=E^{R} \exp \left(-\frac{1}{\hbar} \int_{0}^{T} V\left(X_{t}\right) d t\right)<\infty .
$$

The initial configuration $L_{n}^{Y}(0)=\frac{1}{n} \sum_{i=1}^{n} \delta_{Y_{0}^{i}}$ is almost surely $\delta_{x}$ and, according to (2.4), one observes the mean value of a real function $N$ on the final configuration:

$$
\int_{\mathbb{R}^{d}} N(q) L_{n}^{Y}(T)(d q)=\frac{1}{n} \sum_{i=1}^{n} N\left(Y_{T}^{i}\right) \approx c
$$

which may deviate significantly from the value predicted by the law of large numbers.

Sanov's theorem tells us that the large deviations of $L_{n}^{Y}$ where the $Y_{i}$ 's are identically distributed with the reference law $R^{V}$ is governed by the relative entropy

$$
H\left(P \mid R^{V}\right)= \begin{cases}E^{P}\left(\log \frac{d P}{d R^{V}}\right) & \text { if } P \prec R^{V} \\ +\infty & \text { otherwise }\end{cases}
$$


This approximately means that $\mathbb{P}\left(L_{n}^{Y} \in \mathcal{A}\right) \underset{n \rightarrow \infty}{\asymp} \exp \left[-n \inf _{P \in \mathcal{A}} H\left(P \mid R^{V}\right)\right]$ for $\mathcal{A}$ a subset of $\mathcal{P}(\Omega)$. A precise statement is in terms of a large deviation principle, see [4]. This means that for any closed set $\mathcal{A}, \lim _{\sup _{n \rightarrow \infty}} \frac{1}{n} \log \mathbb{P}\left(L_{n}^{Y} \in\right.$ $\mathcal{A}) \leq-\inf _{P \in \mathcal{A}} H\left(P \mid R^{V}\right)$ and for any open set $\mathcal{A}, \liminf _{n \rightarrow \infty} \frac{1}{n} \log \mathbb{P}\left(L_{n}^{Y} \in \mathcal{A}\right) \geq$ $-\inf _{P \in \mathcal{A}} H\left(P \mid R^{V}\right)$ where $\mathcal{P}(\Omega)$ is endowed with the usual weak topology. With regular enough subsets $\mathcal{A}$ (for instance open convex subsets) and $\mathcal{C}^{\delta}=\{P \in$ $\left.\mathcal{P}(\Omega) ; E^{P}\left[N\left(X_{T}\right)\right] \in[c-\delta, c+\delta]\right\}$ (one introduces $\delta>0$ to make sure that $\left.\mathbb{P}\left(L_{n}^{Y} \in \mathcal{C}^{\delta}\right)>0\right)$, one can expect the following conditional law of large numbers

$$
\lim _{\delta \downarrow 0} \lim _{n \rightarrow \infty} \mathbb{P}\left(L_{n}^{Y} \in \mathcal{A} \mid L_{n}^{Y} \in \mathcal{C}^{\delta}\right)= \begin{cases}1, & \text { if } \mathcal{A} \ni P^{o} \\ 0, & \text { otherwise }\end{cases}
$$

where $P^{o}$ is a minimizer of $P \mapsto H\left(P \mid R^{V}\right)$ on $\mathcal{C}:=\lim _{\delta \downarrow 0} \mathcal{C}^{\delta}=\left\{P \in \mathcal{P}(\Omega) ; E^{P}\left[N\left(X_{T}\right)\right]=\right.$ $c\}$. To see this, remark that (formally)

$$
\mathbb{P}\left(L_{n}^{Y} \in \mathcal{A} \mid L_{n}^{Y} \in \mathcal{C}^{\delta}\right) \underset{n \rightarrow \infty}{\asymp} \exp \left[-n\left(\inf _{P \in \mathcal{A} \cap \mathcal{C}^{\delta}} H\left(P \mid R^{V}\right)-\inf _{P \in \mathcal{C}^{\delta}} H\left(P \mid R^{V}\right)\right)\right] .
$$

Since $H\left(\cdot \mid R^{V}\right)$ is strictly convex and $\mathcal{C}$ is a convex set, $P^{o}$ is unique and this roughly means that conditionally on $L_{n}^{Y} \in \mathcal{C}$, as $n$ tends to infinity $L_{n}^{Y}$ tends to the solution $P^{o}$ of the minimization problem

$$
\text { minimize } H\left(P \mid R^{V}\right) \text { subject to } E^{P}\left[N\left(X_{T}\right)\right]=c
$$

Clearly

$$
\begin{aligned}
H\left(P \mid R^{V}\right) & =E^{P} \log \frac{d P}{d R^{V}}=E^{P} \log \frac{d P}{d R}-E^{P} \log \frac{d R^{V}}{d R} \\
& =H(P \mid R)+E^{P} \frac{1}{\hbar} \int_{0}^{T} V\left(X_{t}\right) d t+\log z_{V}
\end{aligned}
$$

and with Girsanov's theorem one proves that

$$
H(P \mid R)=E^{P} \int_{0}^{T} \frac{1}{2 \hbar}\left|D^{P} X_{t}\right|^{2} d t .
$$

Finally, $P^{o}$ is the critical point of a special functional of type (2.1):

$$
J(P)=\frac{1}{\hbar} E^{P} \int_{0}^{T}\left(\frac{1}{2}\left|D^{P} X_{t}\right|^{2}+V\left(X_{t}\right)\right) d t=H\left(P \mid R^{V}\right)-\log z_{V}
$$

under the constraint $E^{P}\left[N\left(X_{T}\right)\right]=c$, and applying Theorem 2.1 one sees that $\left(P^{o}, \lambda_{o}\right)$ satisfies

$$
\left\{\begin{array}{rll}
D D^{P^{o}} X_{t} & =\nabla V\left(X_{t}\right), \quad 0<t<T ; & \\
X_{0} & =x \\
D^{P^{o}} X_{T} & =\lambda_{o} \nabla N\left(X_{T}\right) & P^{o} \text {-a.s. } \\
E^{P^{o}} N\left(X_{T}\right) & =c
\end{array}\right.
$$


On the other hand, one can characterize $P^{o}$ as the solution of (3.1), using general results about entropy minimization. For each real $\lambda$, let

$$
\Lambda(\lambda):=\log \int_{\mathbb{R}^{d}} e^{\frac{\lambda}{\hbar} N(y)} R_{T}^{V}(d y) \in(-\infty,+\infty] .
$$

The convex conjugate of $\Lambda$ is defined for all real $a$ by $\Lambda^{*}(a):=\sup _{\lambda \in \mathbb{R}}\{a \lambda-\Lambda(\lambda)\} \in$ $(-\infty,+\infty]$.

Theorem 3.1. It is assumed that $N$ satisfies $\Lambda(\lambda)<\infty$ for all $\lambda \in \mathbb{R}$ and that $c$ in (3.1) stands in the interior of the convex hull of the support of $R_{T}^{V}$. Then:

1. There exists a unique solution $P^{o}$ to (3.1) and it is of the form

$$
P^{o}=\exp \left(\frac{\lambda_{o}}{\hbar} N\left(X_{T}\right)-\Lambda\left(\lambda_{o}\right)\right) R^{V}
$$

where $\lambda=\lambda_{o}$ is the unique solution to $\Lambda^{\prime}(\lambda)=c, \lambda \in \mathbb{R}$.

2. $H\left(P^{o} \mid R^{V}\right)=\Lambda^{*}(c)<\infty$.

3. $P^{o}(d \omega)=\int_{\mathbb{R}^{d}} R_{T, y}^{V}(d \omega) \rho^{o}(d y)$ where $\rho^{o}=P_{T}^{o}$ uniquely solves the entropy minimization problem:

$$
\text { minimize } H\left(\rho \mid R_{T}^{V}\right) \text { subject to } \int_{\mathbb{R}^{d}} N(y) \rho(d y)=c, \rho \in \mathcal{P}\left(\mathbb{R}^{d}\right) \text {. }
$$

4. $P^{o}$ is the Markov diffusion associated with (2.2) for the drift

$$
b_{o}(q, t)=\hbar \nabla \log \eta(q, t)
$$

where $\eta$ is the solution to

$$
\left\{\begin{array}{l}
\hbar \partial_{t} \eta=\mathcal{H} \eta, \quad t<T \\
\eta(y, T)=\exp \left(\frac{\lambda_{o}}{\hbar} N(y)-\Lambda\left(\lambda_{o}\right)\right)
\end{array}\right.
$$

and $\mathcal{H}=-\frac{\hbar^{2}}{2} \Delta+V(q)$ is the Hamiltonian defined in (2.12).

5. $P^{o}$ and $\lambda_{o}$ satisfy (3.3).

Idea of proof. Except for the proof of (4), where regularity conditions are left vague, the proof is rigorous.

The interior of the convex hull of the support of $R_{T}^{V}$ is equal to the interior of $\left\{a, \Lambda^{*}(a)<\infty\right\}$ : int dom $\Lambda^{*}$. Since $P^{o}$ solves (3.1), it is known (see [9] for instance) that under the assumption $\Lambda(\lambda)<\infty$ for all $\lambda \in \mathbb{R}$ and $c$ in (3.1) is in $\operatorname{int} \operatorname{dom} \Lambda^{*}, P^{o}$ has the form (3.4). On the other hand, a direct computation shows that $\Lambda^{\prime}\left(\lambda_{o}\right)=\int_{\mathbb{R}^{d}} N d P_{T}^{o}$. This proves (1) and (2).

The proof of (3) is a consequence of the general tensorization formula for the relative entropy:

$$
H\left(P \mid R^{V}\right)=H\left(P_{T} \mid R_{T}^{V}\right)+\int_{\mathbb{R}^{d}} H\left(P_{T, y} \mid R_{T, y}^{V}\right) P_{T}(d y)
$$

Since the given constraint only concerns $P_{T}$ and $H\left(P_{T, y} \mid R_{T, y}^{V}\right)=0$ if and only if $P_{T, y}=R_{T, y}^{V}$, we have $P_{T, y}^{o}=R_{T, y}^{V}$ for $P_{T}^{o}$-a.e. $y$ and $P_{T}^{o}=\rho^{o}$. 
Let us give an idea of the proof of (4). One writes (3.4): $P^{o}=\eta\left(X_{T}, T\right) R^{V}$ with $\eta(y, T)=\exp \left(\frac{\lambda_{o}}{\hbar} N(y)-\Lambda\left(\lambda_{o}\right)\right)$. If the solution $\eta=e^{-\frac{1}{\hbar} S_{L}}$ of $(3.5)$ doesn't vanish we have $\frac{1}{\hbar} V(q)=\eta(q, t)^{-1}\left(\partial_{t}+\frac{\hbar}{2} \Delta\right) \eta(q, t)$ and

$$
\begin{aligned}
T_{t} & :=\eta\left(X_{0}, 0\right)^{-1} \exp \left(-\frac{1}{\hbar} \int_{0}^{t} V\left(X_{s}\right) d s\right) \eta\left(X_{t}, t\right) \\
& =\eta\left(X_{0}, 0\right)^{-1} \exp \left(-\int_{0}^{t} \frac{\left(\partial_{s}+\frac{\hbar}{2} \Delta\right) \eta\left(X_{s}, s\right)}{\eta\left(X_{s}, s\right)} d s\right) \eta\left(X_{t}, t\right) \\
& =\exp -\frac{1}{\hbar}\left(S_{L}\left(X_{t}, t\right)-S_{L}\left(X_{0}, 0\right)-\int_{0}^{t}\left(\partial_{s}+\frac{\hbar}{2} \Delta\right) S_{L}\left(X_{s}, s\right)-\frac{1}{2} \int_{0}^{t}\left|\nabla S_{L}\left(X_{s}, s\right)\right|^{2} d s\right) \\
& =\exp -\frac{1}{\hbar}\left(\int_{0}^{t} \nabla S_{L}\left(X_{s}, s\right) \cdot d X_{s}-\frac{1}{2} \int_{0}^{t}\left|\nabla S_{L}\left(X_{s}, s\right)\right|^{2} d s\right)
\end{aligned}
$$

is a positive local $R$-martingale. If $T$ is not only a local but also a genuine $R$ martingale, then $P^{o}=z_{V}^{-1} \eta(x, 0) T_{T} R$. Now, Girsanov's theorem tells us that the drift of $P^{o}$ is $b^{o}=-\nabla S_{L}$, the desired result.

In particular, at time $T, D^{P^{o}} X_{T}=b^{o}\left(X_{T}, T\right)=\hbar \nabla \log \eta\left(X_{T}, T\right)=\lambda_{o} \nabla N\left(X_{T}\right)$, $P^{o}$-almost surely. Comparing with (3.3), one sees that $\lambda_{o}$ in Theorems 2.1 and 3.1 is the same. This proves (5) and completes the proof of the theorem.

Theorem 3.1 seems to have very little to do with our pathwise line of thought (§2). However, consider the function $S_{L}$ defining the $R$-martingale $T_{t}$ and therefore the Markovian diffusion of (4) in Theorem 3.1.

As a function, since $S_{L}=-\hbar \log \eta$ for $\eta$ a positive solution of Eq. (3.5), $S_{L}$ solves the following Hamilton-Jacobi-Bellman (HJB) equation:

$$
\frac{\partial S_{L}}{\partial t}-\frac{1}{2}\left|\nabla S_{L}\right|^{2}+\frac{\hbar}{2} \Delta S_{L}+V=0 \quad t<T .
$$

Assuming that the solution $S_{L}$ is smooth enough (This is not a trivial assumption since, generically they are not: this is for this equation that the notion of viscosity solution was initially designed: cf [5]), the gradient of HJB provides, since $b_{o}=-\nabla S_{L}$,

$$
D D^{P^{o}} X_{t}=\nabla V\left(X_{t}\right)
$$

This is the a.s Euler-Lagrange equation (3.3), solved by the process critical for the associated stochastic action functional $J(P)$. So the key elements of our pathwise analysis are also present, although implicitly, in the entropic approach.

It has been shown that the study of the symmetries of HJB equation is very rewarding for computations with such critical diffusions (cf [10]).

Let us make a final remark about the product form $(2.16)$. It was the main motivation of Schrödinger's investigation in [12], since it looks like Born's interpretation of the wave function: $\left|\psi_{t}(q)\right|^{2} d q=\psi_{t}(q) \bar{\psi}_{t}(q) d q$ i.e. the probability of presence of a quantum particle when $\psi$ solves Schrödinger's equation for the same Hamiltonian $\mathcal{H}$. 
Formula (3.4): $P^{o}=\eta_{T}\left(X_{T}\right) R^{V}$ which is a direct consequence of the minimization of the relative entropy $H\left(\cdot \mid R^{V}\right)$, can also be given (approximately) a product form

$$
P=\eta_{0}^{*}\left(X_{0}\right) \exp \left(-\frac{1}{\hbar} \int_{0}^{T} V\left(X_{r}\right) d r\right) \eta_{T}\left(X_{T}\right) R_{\alpha}
$$

Here, $R_{\alpha}(d \omega):=\int_{\mathbb{R}^{d}} R_{q}(d \omega) \alpha(d q)$ where $R_{q}$ is the Wiener measure issued from $q$ and $\alpha$ denotes Lebesgue measure. It is an unbounded "reversible" measure of the Wiener process, in the traditional sense of symmetric diffusions (cf [8]). In fact, since it is assumed that $R_{0}^{V}=\delta_{x}, P^{o}$ is only approximately of the form (3.6). Under this $P$, the law $P_{t}$ of $X_{t}$ is given by

$$
\begin{aligned}
P_{t}(d q) & =E^{R_{\alpha}}\left[\eta_{0}^{*}\left(X_{0}\right) \exp \left(-\frac{1}{\hbar} \int_{0}^{T} V\left(X_{r}\right) d r\right) \eta_{T}\left(X_{T}\right) \mid X_{t}=q\right] d q \\
& =\eta^{*}(q, t) \eta(q, t) d q
\end{aligned}
$$

where we used the Markov property of $R_{\alpha}$ at time $t, 0<t<T$, and we have introduced

$$
\begin{aligned}
\eta^{*}(q, t) & =E^{R_{\alpha}}\left[\eta_{0}^{*}\left(X_{0}\right) \exp \left(-\frac{1}{\hbar} \int_{0}^{t} V\left(X_{r}\right) d r\right) \mid X_{t}=q\right] \\
\eta(q, t) & =E^{R_{\alpha}}\left[\exp \left(-\frac{1}{\hbar} \int_{t}^{T} V\left(X_{r}\right) d r\right) \eta_{T}\left(X_{T}\right) \mid X_{t}=q\right] .
\end{aligned}
$$

Using forward and backward exponential martingales, one shows that

$$
\left\{\begin{array} { l } 
{ \hbar \partial _ { t } \eta ( q , t ) = \mathcal { H } \eta ( q , t ) } \\
{ \eta ( \cdot , 0 ) = \eta _ { 0 } }
\end{array} \quad \text { and } \quad \left\{\begin{array}{l}
-\hbar \partial_{t} \eta^{*}(q, t)=\mathcal{H} \eta^{*}(q, t) \\
\eta^{*}(\cdot, 0)=\eta_{0}^{*}
\end{array} .\right.\right.
$$

Given the fact that $\eta$ and $\eta^{*}$ can be regarded as time reversed of each other, the product form of $P_{t}(d q)$ is, therefore, the expression of a time dependent version of reversibility generalizing considerably the above-mentioned notion of symmetric diffusions. Explored since the mid-eighties (cf [13], [3], etc ...), this idea is the basic one in the program of stochastic deformation whose simplest variational problem with constraint was considered here.

\section{References}

[1] K.L. Chung and J.C. Zambrini. Introduction to Random Time and Quantum Randomness. World Scientific, 2003.

[2] A.B. Cruzeiro, L. Wu, and J.C. Zambrini. Bernstein processes associated with a Markov process. In R. Rebolledo, editor, Stochastic analysis and mathematical physics, ANESTOC'98. Proceedings of the Third International Workshop, Trends in Mathematics, pages 41-71, Boston, 2000. Birkhäuser.

[3] A.B. Cruzeiro and J.C. Zambrini. Malliavin calculus and Euclidean quantum mechanics, I. J. Funct. Anal., 96(1):62-95, 1991. 
[4] A. Dembo and O. Zeitouni. Large Deviations Techniques and Applications. Second edition. Applications of Mathematics 38. Springer Verlag, 1998.

[5] W.H. Fleming and H.M. Soner. Controlled Markov Processes and Viscosity Solutions, volume 25 of Applications of Mathematics. Springer, 1993.

[6] H. Föllmer. Random fields and diffusion processes, in Ecole d'Eté de Probabilités de Saint-Flour XV-XVII-1985-87, volume 1362 of Lecture Notes in Mathematics. Springer, Berlin, 1988.

[7] M. Giaquinta and S. Hildebrandt. Calculus of variations I, volume 310 of Grund. der math. Wissensch. Springer, 1996.

[8] N. Ikeda and S. Watanabe. Stochastic Differential Equations and Diffusion Processes. North Holland, 1981.

[9] C. Léonard. Minimizers of energy functionals. Acta Math. Hungar., 93(4):281-325, 2001.

[10] P. Lescot and J.C. Zambrini. Probabilistic deformation of contact geometry, diffusion processes and their quadrature. In Seminar on Stochastic Analysis, Random Fields and Appplications V, Eds. R. Dalang, M. Dozzi, F. Russo, Progress in Probability Series, Birkhäuser, 2008.

[11] N. Privault and J.C. Zambrini. Markovian bridges and reversible diffusions with jumps. Ann. Inst. H. Poincaré Probab. Statist., 40:599-633, 2004.

[12] E. Schrödinger. Sur la théorie relativiste de l'électron et l'interprétation de la mécanique quantique. Ann. Inst. H. Poincaré, 2:269-310, 1932. Available at http://archive.numdam.org/ARCHIVE/AIHP/

[13] J.C. Zambrini. Variational processes and stochastic versions of mechanics. J. Math. Phys., 27(9):2307, 1986.

[14] J.C. Zambrini. From the geometry of parabolic PDE to the geometry of SDE. In A.B. Cruzeiro N. Obata, H. Ouerdiane, editor, Mathematical Analysis of Random Phenomena. World Scientific, 2007.

\section{Acknowledgment}

The second author is grateful to Ascona's organization's team for this new opportunity to enjoy their classical meeting.

Christian Léonard

Modal-X. Université Paris Ouest. Bât. G, 200 av. de la République. 92001 Nanterre, France

e-mail: Christian.Leonard@u-paris10.fr

Jean-Claude Zambrini

GFMUL, Av. Prof. Gama Pinto 2. 1649-003 Lisbon, Portugal

e-mail: zambrini@cii.fc.ul.pt 